\title{
Silencing of miR-20b-5p Exerts Inhibitory Effect on Diabetic Retinopathy via Inactivation of THBSI Gene Induced VEGF/Akt/PI3K Pathway
}

This article was published in the following Dove Press journal: Diabetes, Metabolic Syndrome and Obesity: Targets and Therapy

\author{
YanBo Ma' \\ ChunYing Dong ${ }^{2}$ \\ XiHui Chen' \\ RuiXi Zhu' \\ Jie Wang (D)
}

'Department of Ophthalmology, Heilongjiang Provincial Hospital, Harbin, Heilongjiang, I 50036, People's Republic of China; ${ }^{2}$ Department of Infectious Disease, Heilongjiang Provincial Hospital, Harbin, Heilongjiang, I50036, People's Republic of China
Correspondence: Jie Wang Department of Ophthalmology, Heilongjiang Provincial Hospital, Harbin, Heilongjiang, I50036, People's Republic of China

Tel/Fax +86- I3656838933

Email Jiewang8I@hotmail.com
Introduction: Diabetic retinopathy (DR) is a damaging complication of the eye. Studies investigating molecular mechanisms of DR are lacking, leading to poor clinical outcomes. miR-20b-5p is up-regulated in DR. The present study aimed to confirm the involvement of miR-20b-5p in DR and the mechanism involved.

Methods: Microarray analysis was done to study the differentially expressed miRs. DR model was established using Sprague-Dawley rats, the expression of miR-20b-5p was altered using inhibitor or mimic as treatment. THBS1 was one of the potential genes identified by microarray bioinformatics analysis associated with DR. The expression of THBS1 was suppressed by siRNA to study the mechanism behind involvement of miR-20b-5p in DR. In addition, the levels of miR-20b-5p VEGF/PI3K/Akt pathway associated genes were studied. Correlation between THBS1 and miR-20b-5p was evaluated. Cell apoptosis, growth and tube formation assay was performed.

Results: The retinal tissues of DR rats showed over-expressed miR-20b-5p and decreased THBS1 via VEGF/PI3K/Akt cascade. THBS1 was confirmed as the target gene of miR-20b$5 \mathrm{p}$ by dual-luciferase reporter gene assay. Upregulation of miR-20b-5p or knockdown of THBS1 caused increased tube formation and cell proliferation, whereas it blocked the cell apoptosis of endothelial cells in rats.

Conclusion: The outcomes suggested that silencing of miR-20b-5p resulted in inhibition of tube formation and cell growth in vascular endothelial cells of rats subjected to DR altering the VEGF/PI3K/Akt cascade by up-regulation of THBS1.

Keywords: diabetic retinopathy, miR-20b-5p, VEGF/PI3K/Akt, endothelial cells

\section{Introduction}

Cases of diabetes are on the rise globally, with over 150 million patients in China. Diabetes leads to numerous complications in the body, diabetic retinopathy (DR) is one of them, which leads to partial or permanent loss of vision. ${ }^{1-3}$ DR is a microvascular disorder which is activated by leakage of retinal capillary due to hyperglycemic condition of diabetic subjects. Earlier studies have suggested that oxidative stress mediated injury of retinal tissue contributed heavily to development of DR. ${ }^{4}$ However, more studies are needed to understand the molecular and cellular mechanisms involved in development and progression of DR to improve its management. ${ }^{5}$

MicroRNAs (miRs) are considered to be important regulators of various processes in the body and are also involved in regulatory function of retina by altering 
expression of various genes. ${ }^{6}$ Reports have emerged which suggested involvement of miRs in development of DR which was evidenced by variations in expression of miRs under diabetic condition in DR. ${ }^{7-10}$ MiR-365 has been reported to promote DR via suppressing Timp3. ${ }^{7}$ Inhibition of circRNA DMNT3B promotes diabetic retinal vascular dysfunction involving miR-20b-5p. ${ }^{8}$ In an earlier study, miR-20b-5p was reported to be significantly upregulated in DR. ${ }^{11}$ In addition to this, the expression of miR-20b-5p is altered in various malignancies such as stomach, ${ }^{12}$ blood, ${ }^{13}$ breast, ${ }^{14}$ and colorectal cancer. ${ }^{15}$ MiR-20b-5p is reported to target the $3^{\prime}$ UTR of BTG3 in non-small cell lung cancer. ${ }^{16}$ Thrombospondin 1 (THBS1) is an antiproliferative gene, over-expression of this gene could be triggered by suppression of PI3K, i.e, phosphatidylinositol $3^{\prime}$-kinase. ${ }^{17}$ The PI3K/Akt cascade is associated with formation of blood vessels, migration and proliferation of endothelial cells; PI3K was also reported to induce endothelial growth factor (VEGF). ${ }^{18}$ It was reported that VEGF is involved in regulation of angiogenesis of ocular cells in DR which includes the process of tube formation, migration of vascular endothelial cells and their proliferation. ${ }^{19}$ Interestingly, in a study, the VEGF/ $\mathrm{PI} 3 \mathrm{~K} / \mathrm{Akt}$ axis was found to be involved in angiogenesis of retinal cells. ${ }^{20}$

Based on these studies, the present work presupposes that miR-20b-5p could be associated with DR by involving the VEGF/PI3K/Akt cascade specifically involving the THBS1 gene. The present study aimed to develop and establish the rat model of DR and to confirm the involvement of miR-20b-5p in DR.

\section{Methods}

\section{Animal Studies}

For the study, Sprague-Dawley (SD) rats $(n=75)$ aged between 8 to 10 weeks and weighing between 210-235 $\mathrm{g}$ were used. The animal studies received approval from the animal ethics committee of Heilongjiang Provincial Hospital, Harbin, Heilongjiang 150,036, China. The experiments were done in accordance with Animal Research: Reporting of In Vivo Experiments (ARRIVE) guidelines. For welfare of animals we followed Chinese National Guidelines (GB/T 35,892-20,181) for the ethical review of laboratory animal welfare. The rats were divided randomly into DR group $(n=30)$ and normal rats $(n=45)$. The diabetes was induced in rats by injecting Streptozotocin $60 \mathrm{mg} / \mathrm{kg}$ (STZ) $(0.01 \mathrm{M} / 1$, in citrate buffer
$\mathrm{pH}$ 4.4). ${ }^{21}$ The normal rats were treated with vehicle (citrate buffer $0.01 \mathrm{M} / 1, \mathrm{pH} 4.4$ ). The animals, after these treatments, were housed in room temperature conditions with free access to water and pellet diet. After the rats were injected with STZ, the blood was collected after 72 hours for determining blood glucose levels (BGC). The rats were confirmed to be diabetic if the BGC levels were greater than $16.7 \mathrm{mM} / \mathrm{l}$. The rat model of diabetes was confirmed when the blood BGC levels after 4 weeks were higher than $16.7 \mathrm{mM} / \mathrm{l}^{22}$ After 12 weeks, the diabetic rats were studied for electro-physiological measurement of retinal tissues. The diabetic rats were confirmed as having retinopathy by measuring the Ops wave and incubation period of F-ERG. The DR and normal rats, after the study, were euthanized and eyeballs were removed. The harvested eyeballs were fixed in paraformaldehyde (4\%) and fixed in paraffin and were submitted to rotary microtome to obtain sections. Some of the eyeballs were also stored in liquid nitrogen at $-80^{\circ} \mathrm{C}$ for isolating total RNA and protein content.

\section{Microarray-Based Bioinformatics Analysis for Gene Expression of DR}

Microarray analysis was done to study the gene expression in DR rats, the differentially expressed genes were studied using various gene databases. For microarray analysis, various databases were searched; TargetScan (http:// www.targetscan.org/vert 72/) was explored to establish the relationship between differentially expressed genes, Chilibot and DisGeNET data base were explored to study various genes, proteins or ligands. ${ }^{23,24}$

\section{Reverse Transcription Quantitative Polymerase Chain Reaction (RT-qPCR)}

The isolated retinal tissue and transfected cells were processed to extract total RNA with the help of RNeasy isolation kit. The primers used for RT-qPCR were as follows, PI3K: Forward 5'TTAAACGCGAAGGCAACGA-3' Reverse 5'-CAGTCT CCTCCTGCTGTCGAT-3', Akt: Forward5'-ACTCATTCC AGACCCACGAC-3', Reverse 5'- CCGGTACACCA CGTTCTTCT- 3', VEGF:Forward5'-ATCATGCGGATCA AACCTCACC-3', Reverse5'-GGTCTGCATTCATCTGC TAT-3', CD34: Forward 5'-GCAAGCCACCAGAGCTATTC - 3', Reverse 5'-GGTCCCAGGTCCTGAGCTAT-3', eNOS: Forward 5'-CCAGCTAGCCAAAGTCACCAT-3', Reverse 5'-GTCTCGGAGCCATACAGGATT-3', Actin: Forward 5'CAGCCTTCCTTCCTGGGTATG-3', Reverse 5'- TAG 
AGCCACCAATCCCACG- 3'. The isolated RNA was reverse transcribed into cDNA using Prime Script-RT, as per the supplied instructions. The reaction conditions were also as per provided instructions. The reaction solution was then submitted for RT-qPCR with the help of Premix Ex TaqTM II Kit (Takara Biotech, CAT \#: RR820B), for the experiment, actin and U6 were selected as loading controls for target genes. The RT-qPCR was done with the help of RT-qPCR system (Biocompare USA), the reaction conditions were as follows: denaturation for 15 minutes at $95^{\circ} \mathrm{C}$, total 40 cycles of denaturation for 15 seconds, extension and annealing for $60 \mathrm{sec}-$ onds at $60^{\circ} \mathrm{C}$. The detection of miR-20b-5p and mRNA levels of Akt, CD34, VEGF, PI3K, and THBS1 were calculated.

\section{Western Blot Analysis}

The expression of proteins was done in retinal tissues which were homogenized prior to analysis. The tissue homogenates were lysed using RIPA lysis buffer and the resultant was centrifuged at $5000 \mathrm{~g}$ for 20 minutes at $4^{\circ} \mathrm{C}$ using a cooling centrifuge. The supernatant was processed and total proteins were evaluated with protein estimation kit. The proteins were solubilized in buffer and heated to boiling for 5 minutes, after cooling the proteins were centrifuged, the proteins were loaded on SDS-PAGE $(10 \%)$, and blotted on nitrocellulose membranes. The membranes were blocked using skimmed milk (5\%) for 1 hour and the membranes were incubated with $\mathrm{I}^{\text {ry }}$ antibodies at $4^{\circ} \mathrm{C}$ for 12 hours. The blots were analyzed using Image processing software. Actin was selected as loading control, the expression of proteins was standardized against control.

\section{Construction of Plasmid}

Genome was used a template, DNA oligo and siRNA of overexpressing RNA were prepared using the target sequences which interfered with the expression of miR20b-5p, at last a double stranded DNA was obtained. The ends of oligonucleotides were inserted with EcoR1 and Age1 restriction sites. The primers used were miR-20b-5p mimic: 5'-CCTAGTAGTGCCAAAGTGCT-3', miR-20b-5p inhibitor: 5'-CCAGGAGTACTAGAAGTGATCA-3' and siRNATHBS1: 5'-GCTCCAGCTCTACCAGTGTC-3'.

\section{Transfection of Cells}

The eye balls of DR rats were processed to separate the vitreous and retinal tissues after removing the anterior segment tissues. The retinal tissues were further subjected to homogenization and the homogenates were subjected to trypsinization with a trypsin buffer $(2 \%)$ for 30 minutes and then centrifuged followed by incubation with collagenase-II for 20 minutes and filtered. The resultant was mixed with culture media and seeded with fibronectin and maintained at room temperature with $5 \% \mathrm{CO} 2$. The vascular endothelial cells were seeded into six well plates and once they reached confluence of $30-50 \%$, they were transfected using Lipofectamine-2000 transfecting reagent. The cells were transfected with miR-20b-5p mimic, miR-20b-5p inhibitor, miR-20b-5p inhibitor+siRNA-THBS1, siRNA- THBS1 and NC plasmids. The cells were incubated with serum-free OptiMEM for 5 minutes at $37^{\circ} \mathrm{C}$. The cells were seeded and maintained at $37^{\circ} \mathrm{C}$ in $5 \% \mathrm{CO} 2$ for 7 hours. The culture media were replaced with complete media and incubated for 48 hours. The cells were divided into normal control, blank (non-transfected), Negative control (non-sense sequence transfected cells), miR-20b-5p mimic transfected cells, miR-20b-5p inhibitor transfected cells, siRNA- THBS1 transfected cells, Negative control mimic transfected cells and miR-20b-5p inhibitor+siRNA- THBS1 treated cells and miR-inhibitor + siRNA- THBS1 transfected cells.

\section{Dual-Luciferase Reporter Gene Assay}

Based on microarray analysis, we speculated that THBS1 was a target gene, and hence wanted to evaluate whether miR-20b-5p regulated the gene THBS1 via binding to the 3'UTR region, thus we performed dual luciferase reporter assay to verify THBS1 was the direct target of miR-20b-5p. Looking into the binding site sequences of the 3'UTR of miR-20b-5p and THBS1, the wild type THBS1 with THBS1-3'UTR along with the mutant sequence of miR20b-5p and THBS1-3'UTR (Mut-BYG1) were produced. The wild type and mutant with the right sequences were cotransfected with miR-20b-5p inhibitor in the endothelial cells respectively. The cells were submitted to lysis after 48 hours of transfection. The cells were rinsed using phosphate buffered saline thrice. The cells were submitted to lysis buffer, the supernatant was transferred to 96 well plate followed by dual luciferase assay.

\section{The MTT Assay}

The vascular endothelial cells were treated with RPMI-1640 media loaded with fetal bovine serum (FBS) (10\%) to prepare cell suspension $\left(5 \times 10^{4}\right.$ cells $\left./ \mathrm{mL}\right)$. The cells were incubated in 96 well plates at room temperature under 5\% $\mathrm{CO} 2$ conditions. After incubating for 24,48 , and 72 hours, each well received $10 \mu \mathrm{L}$ of 3-(4, 5-dimethylthiazol-2-yl)2,5-diphenyltetrazolium bromide (MTT) and was incubated 
for 4 hours. The absorption of each well was recorded at 570 $\mathrm{nm}$ with the help of MTT assay recorder (BioRad).

\section{Tube Formation Assay}

Matrigel $(70 \mu \mathrm{L})$ was added to each well of 96 well plate, and the wells were incubated at room temperature for 1 hour for solidification. The cells were lysed and treated with $0.05 \%$ trypsin and rinsed with culture media three times to remove the leftover traces of serum and were then counted. After this, $5 \times 10^{5}$ cells were transferred to each well followed by addition of matrigel $100 \mu \mathrm{L}$ to each well. The 96 well plate was incubated for 15 hours at room temperature and the cells were observed under a microscope. The length of each tube was measured with the help of image analyzing software Image-ProPlus (Media Cybernetics USA).

\section{Flow Cytometry Studies}

The cells were transfected and were then treated with solution of trypsin and EDTA and then centrifuged, the cells were rinsed with cold PBS. The Annexin-V-FITC kit was used; cells approximately $1 \times 10^{6}$ were mixed with $100 \mu \mathrm{L}$ of Annexin-V/FITC/PI reagent and were incubated for 15 minutes at $37^{\circ} \mathrm{C}$. The FITC and PI analysis were done at $490 \mathrm{~nm}$ for determining cell apoptosis.

\section{Determination of Reactive Oxygen Species (ROS)}

The retinal tissues were rinsed with PBS, homogenized, and incubated with H2DCFDA $(20 \mu \mathrm{M})$ for 5 hours. The cells were centrifuged at $5000 \mathrm{~g}$ for 10 minutes; about 100 $\mu \mathrm{L}$ suspension was transferred to 96 well plates. The samples were analyzed using a Fluorescence spectrophotometer at excitation and emission coefficient of $485 \mathrm{~nm}$ and $525 \mathrm{~nm}$ respectively. The readings were recorded and outcomes were obtained as fluorescence/microgram of protein and presented as \% control.

\section{Statistical Analysis}

All the statistics were performed on GraphPad prism software. The results were presented as mean \pm standard deviation. The groups were compared by Student's $t$-test and multiple groups were compared by one way ANOVA. Values of $\mathrm{P}<0.05$ were considered significant.

\section{Results}

miR-20b-5p Regulates the Expression of BTGI Involving VEGF/Akt/PI3K Cascade in Diabetic Retinopathy

We screened the diabetes-related gene expression chip GSE7014 with the help of R language, to screen the differentially expressed genes we used the $|\operatorname{LogFC}|>2$ and $\mathrm{P}<0.05$. Microarray analysis employing databases such as TargetScan, Chilibot and DisGeNET assisted and sorted about 7 differentially expressed genes (Figure 1A) (LOC402560, VEGFA, ENC1, THBS1, PKP2, S100A6 andSORBS3), which were further analyzed. We further used Chilibot database for establishing relation between genes which are differentially expressed in diabetes, the database suggested PKP2, S100A6 and THBS1 were interrelated with diabetes. We again utilized DisGeNet database for screening potential DR-related genes, the database confirmed ENC1 and SORB3 genes also associated with diabetes. An earlier study had confirmed THBS1 as the potential gene associated with the liver. ${ }^{25}$ The heat map analysis suggested that expression of THBS1 was significantly lower in the retinal tissues of diabetes group compared to control group. In the study, to further explore the possible molecular mechanism associated with THBS1, TargetScan, DIANA and miRSearch analyses were done for prediction of miR which could regulate the gene THBS1. TargetScan, DIANA and miRSearchformiRs predicted about 10, 9 and 11 miRs responsible for regulating THBS1, among them miR-20b-5p was present in prediction data of all the three databases (Figure 1B). In the present work we focused on miR-20b-5p based on its role in development and progression of DR via THBS1 mediated by VEGF/Akt/PI3K cascade.

\section{miR-20b-5p Was Over-Expressed in DR, THBSI Was Suppressed via Activation of VEGF/Akt/PI3K Cascade}

We studied the expression levels of miR-20b-5p, THBS1, VEGF, PI3K, Akt, eNOS and CD34 in the retinal tissues of DR rats with the help of RT-qPCR and Western blot assay. The outcomes of RT-qPCR (Figure 2A) suggested that expression of miR-20b-5p was significantly increased, whereas the mRNA THBS1 levels were significantly lower in retinal tissues of DR rats compared to normal rats. The mRNA levels of VEGF/Akt/PI3K cascade associated genes eNOS and CD34 were over-expressed significantly in DR 
A

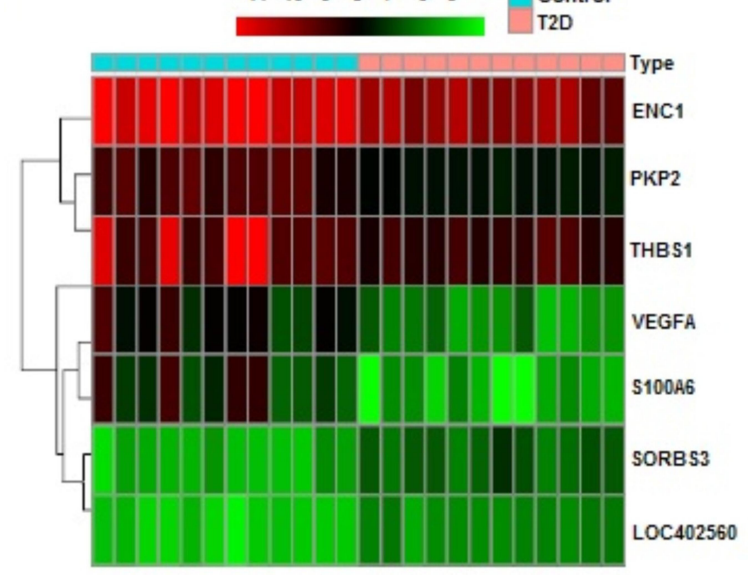

B

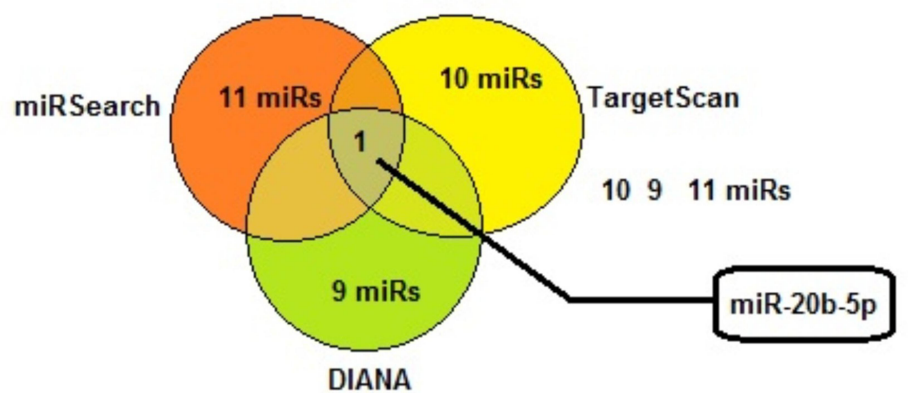

Figure I miR-20b-5p may be involved in regulating the Thrombospondin I gene mediated VEGF/Akt/PI3K pathway in diabetic retinopathy. (A) Screening results of diabetesrelated gene expression chip GSE70I4 for differentially expressed genes, thermal map showing 7 differentially expressed genes (LOC402560, VEGFA, ENCI, THBSI, PKP2, SI00A6 and SORBS3). (B) miR-20b-5p emerged to be the common miRNA present in TargetScan, DIANA, and miRSearch which may target THBSI.
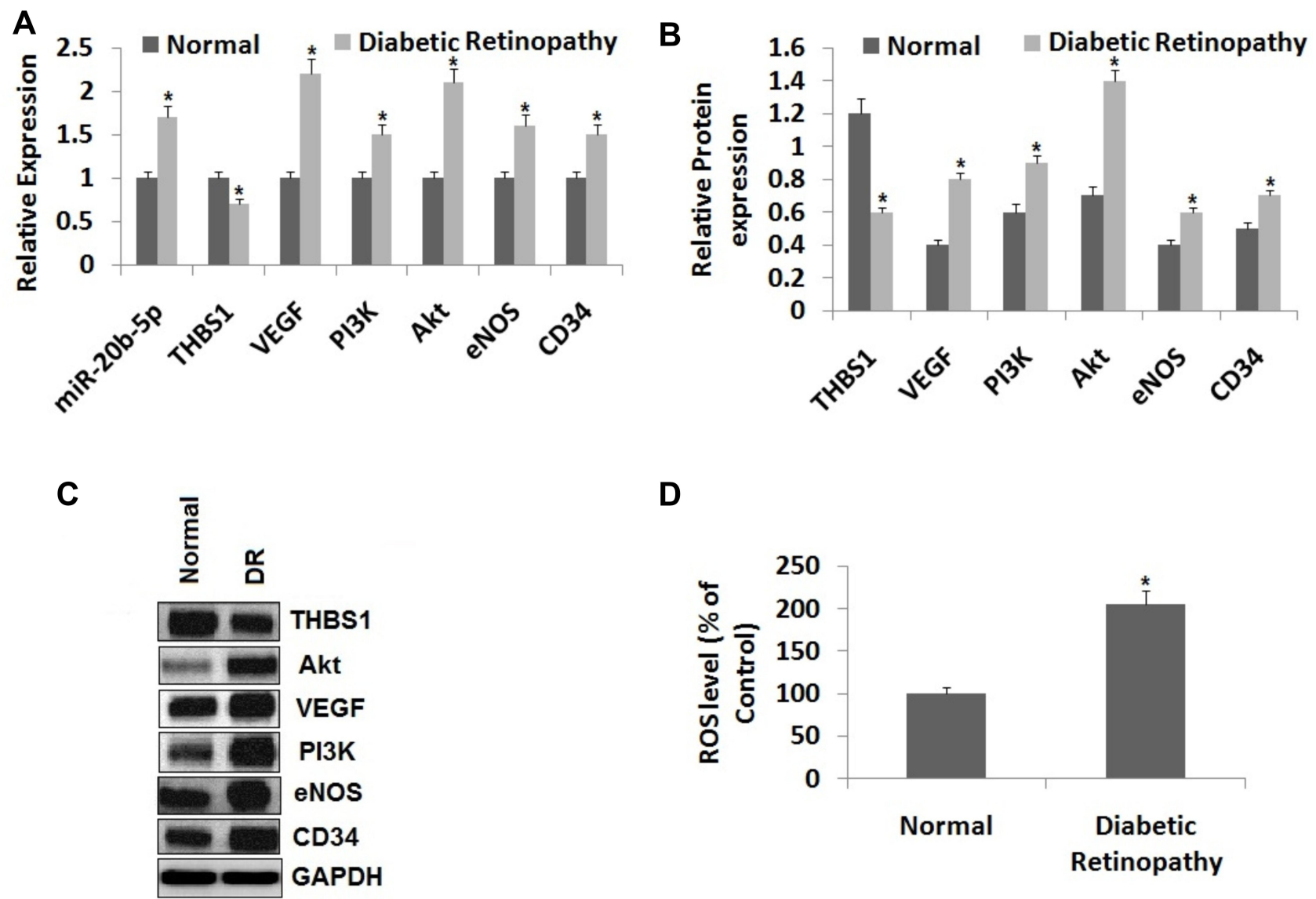

D

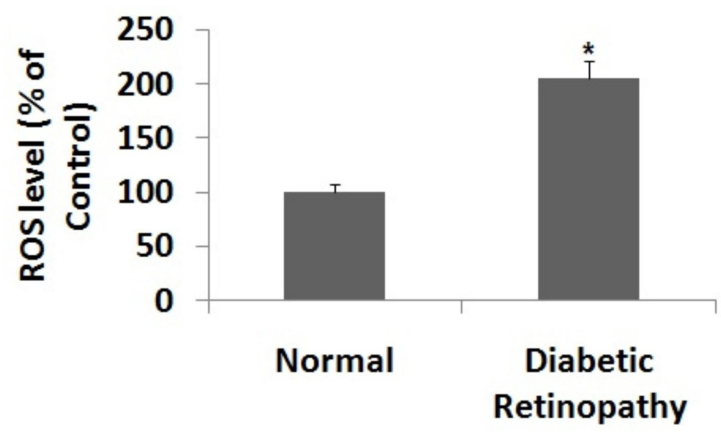

Figure 2 miR-20b-5p and VEGF/Akt/PI3K pathway are over-expressed, whereas the expression of THBSI is suppressed in the retinal tissue of diabetic retinopathy rats. (A) Expression of miR-20b-5p and mRNA levels of VEGF/Akt/PI3K signaling pathway associated genes in the retina of diabetic retinopathy rat tissues and normal retinal tissues. $(\mathbf{B}, \mathbf{C})$ Western blot analysis for expression of proteins of VEGF/Akt/PI3K pathway associated genes. (D) Levels of reactive oxygen species (ROS) in retinal tissues of diabetic retinopathy and normal rats. ${ }^{*} \mathrm{P}<0.05$ compared to retinal tissue of normal rats. The results are mean \pm standard deviation; sample $t$-test was done for statistical significance. 
retinal tissues compared to the normal rats. In addition to this, Western blot assay (Figure 2B and C) suggested that THBS1 protein levels were decreased significantly whereas CD34 and eNOS, which are the VEGF/Akt/PI3K cascade related proteins, were significantly over-expressed in DR rats' retinal tissues compared to normal rats. Also, the ROS levels were overexpressed in DR retinal tissues of rats compared to normal (Figure 2D). These findings suggested that miR20b-5p, CD34 and eNOS were overexpressed in retinal tissue of diabetic rats whereas THBS1 was suppressed.

\section{THBSI Was the Potential Target of miR-20b-5p}

THBS1 was identified as a potential target of miR-20b-5p with the help of in silico target prediction software and luciferase activity assay. The bioinformatics analysis by TargetScan suggested that THBS1 had target binding site for miR-20b-5p (Figure 3A). To further verifywhether miR-20b-5p regulated THBS1, luciferase assay was done for which the wild type (WT) THBS1 (WT-THBS1) with the THBS1 3'UTR was prepared also mutant vector miR-20b-5p combined with seed- sequence of THBS1 $3^{\prime} \mathrm{UTR}$ region. The endothelial cells of DR rats with THBS1 3 'UTR-WT showed significant decrease in luciferase activity when transfected with miR-20b-5p inhibitor when compared to cells co-transfected with NC. The cells with THBS1 $3^{\prime}$ UTR-MUT showed similar activity to that of miR-20b-5p inhibitor and negative control treated cells (Figure 3B). These outcomes suggested that THBS1 was the favorable target of miR-20b-5p.

\section{Suppression of miR-20b-5p Leads to Over-Expression of THBSI and Blocks the VEGF/Akt/PI3K Cascade in Diabetic Retinopathy Rats}

To analyze the regulatory effect of miR-20b-5p on expression of THBS1 and VEGF/Akt/PI3K cascade we performed RT-qPCR for mRNA levels and immunoblotting analysis for protein levels post-transfection in endothelial cells of diabetic retinopathy rats. The results (Figure 4) suggested that the expression of CD34 and eNOS (the VEGF/Akt/PI3K related genes) were parallel to blank group, negative control and miR-20b-5p inhibitor+siRNA-THBS1 group. Also, the

A
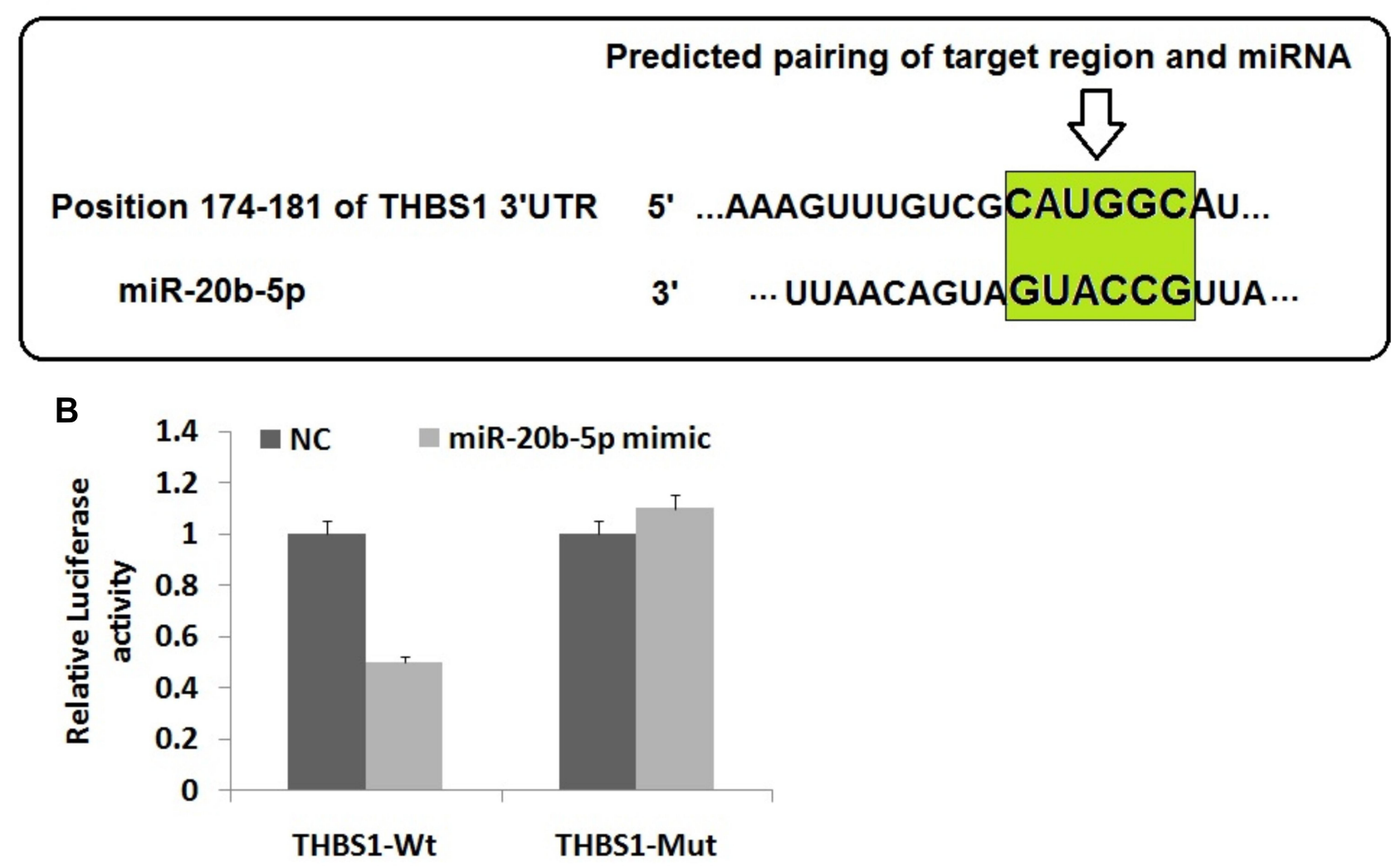

Figure 3 Thrombospondin I (THBSI) gene is target gene of miR-20b-5p. (A) The THBSI gene has potential binding site between the 3'UTR region of THBSI and miR-20b$5 p$ as predicted in TargetScan. (B) Results of Luciferase assay post-transfection with negative control and miR-20b-5p mimic. The results are mean \pm standard deviation. 

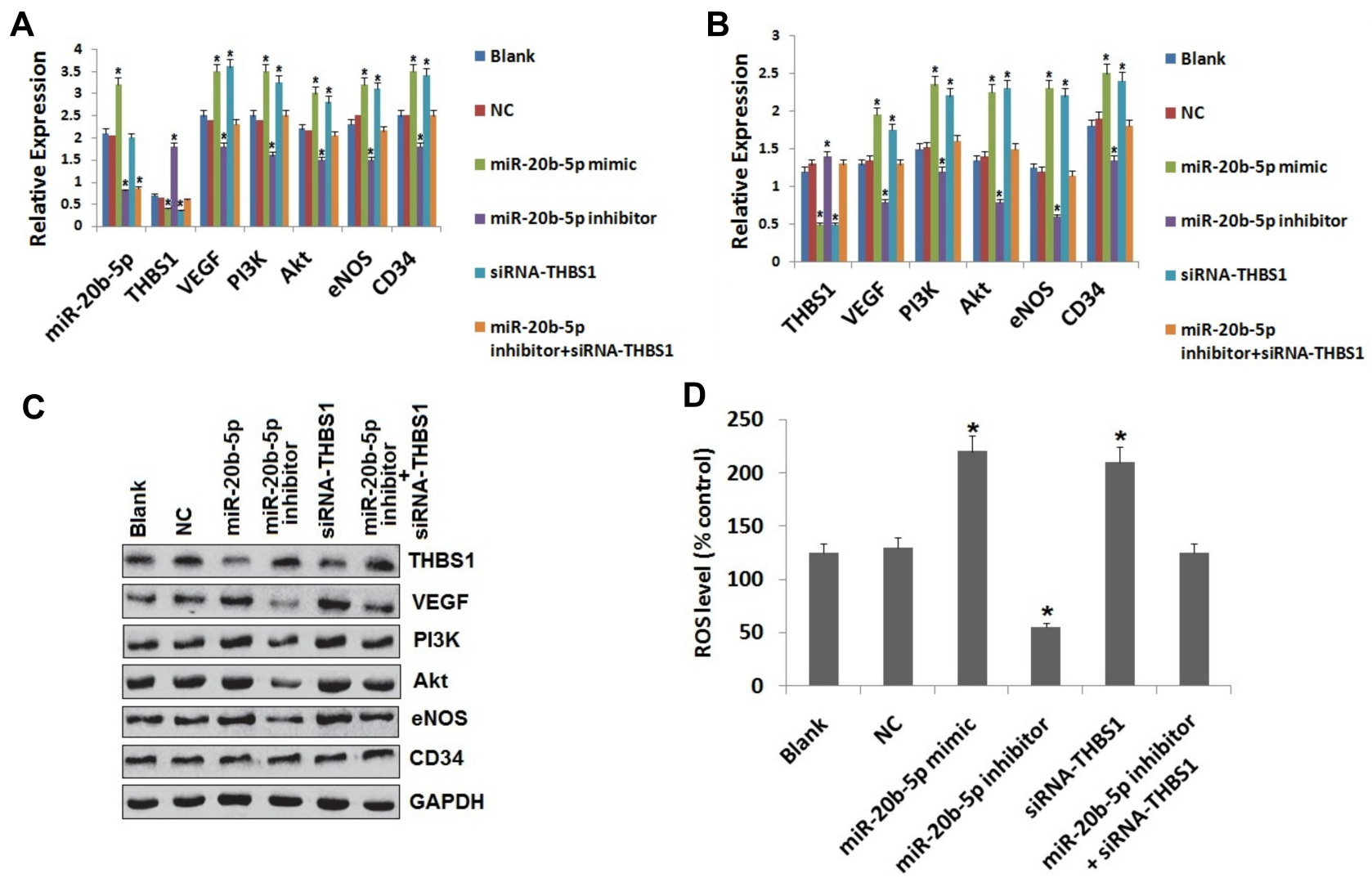

Figure 4 Up-regulated miR-20b-5p activates the VEGF/Akt/PI3K signaling cascade by suppressing the THBSI in the vascular endothelial cells in DR rats. (A) miR-20b-5p levels and mRNA levels of VEGF/Akt/PI3K associated genes in the retinal tissues of diabetic retinopathy rats post-transfection. (B) Protein levels of VEGF/Akt/PI3K related genes in retinal tissue of DR rats post-transfection. (C) Western blot analysis for expression of VEGF/Akt/PI3K associated genes post-transfection. (D) Levels of reactive oxygen species (ROS) in the cells post-transfection. $* \mathrm{P}<0.05$ compared to blank group rats. The results are mean \pm standard deviation.

expression of miR-20b-5p in siRNA+THBS1 treated group was similar to blank treatment. The miR-20b-5p inhibitor group, when compared to blank group, had decreased expression of miR-20b-5p along with decreased expression of eNOS and CD34 (VEGF/Akt/PI3K related proteins) along with ROS with exception of overexpressed THBS1. We also concluded that overexpression of miR-20b-5p activated the $\mathrm{VEGF} / \mathrm{Akt} / \mathrm{PI} 3 \mathrm{~K}$ signaling cascade via its negative regulatory effect on THBS1.

\section{Inhibition of miR-20b-5p or Over-Expression of THBSI and Modulates the Proliferation of Endothelial Cells of Diabetic Retinopathy Rats}

We further studied the consequences of miR-20b-5p and THBS1 on process of proliferation of endothelial cells with the help of MTT assay. The outcomes (Figure 5) suggested that cell viability in blank group, NC and miR20b-5p inhibitor+siRNA-THBS1 group was similar.
However, miR-20b-5p mimic group and siRNA-THBS1 group, in comparison to blank group, had higher optical density at 48 hours and 72 hours, suggesting increased cell viability. Also, the miR-20b-5p inhibitor group showed decreased optical density post 48 and 72 hours against the blank group, suggesting decreased cell viability. The outcomes indicated that over-expression of miR-20b-5p could increase the cell viability in diabetic rats while suppression of miR-20b-5p showed inverse effects. Interestingly, siRNA-THBS1 disabled the suppressing effect of miR$20 b-5 p$ inhibitor on the viability of endothelial cells.

\section{Suppression of miR-20b-5p or} Over-Expression of THBSI Could Decrease Tube Formation in Diabetic Retinopathy Rats

To study whether miR-20b-5p and THBS1 regulated the tube formation in retinal cells of diabetic retinopathy rats, we performed tube formation capability assay. The results 


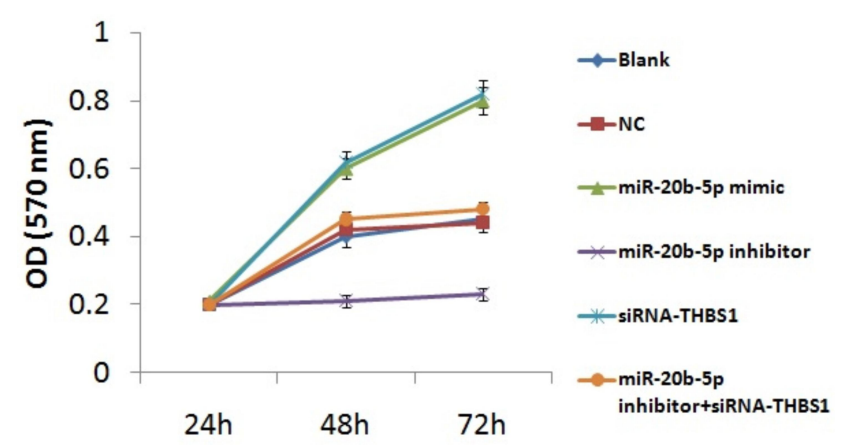

Figure 5 Over-expression of miR-20b-5p or suppression of Thrombospondin I (THBSI) gene promotes proliferation of endothelial cells of diabetic retinopathy rats. The results are mean \pm standard deviation.

(Figure 6A and B) suggested that tube formation results in blank, NC and miR-20b-5p inhibitor+siRNA-miR-20b-5p group did not vary. However, the process of tube formation was triggered in miR-20b-5p mimic and siRNATHBS1 group and suppressed in miR-20b-5p inhibitor group against blank. Outcomes suggested that miR-20b$5 \mathrm{p}$ over-expression increased tube formation ability in cells of diabetic retinopathy rats, whereas inverse effects were observed upon inhibition of miR-20b-5p. Also, siRNA-THBS1 disabled the suppressive effect of miR20b-5p-inhibitor on tube formation capacity of cells.

\section{Suppression of miR-20b-5p or Over-Expression of THBSI Increases the Apoptotic Cells in Diabetic Retinopathy Rats} Flow cytometry study was carried out to determine the role of miR-20b-5p and THBS1 in cell apoptosis. The outcomes (Figure 7) suggested that apoptosis in cells did not differ in blank, NC and miR-20b-5p inhibitor+siRNATHBS1 treated group. Compared to blank, the apoptosis in cells decreased in miR-20b-5p-mimic group and siRNATHBS1 treated rats, whereas it increased in cells treated with miR-20b-5p-inhibitor. These outcomes suggested that over-expression of miR-20b-5p decreased cell apoptosis in diabetic retinopathy rats, whereas miR-20b-5p inhibitor showed opposite effects. The siRNA-THBS1 was found to show counteracting effect on the inhibitory effects or miR-20b-5p inhibitor on endothelial cells on apoptosis.

\section{Discussion}

Diabetic retinopathy is a metabolic disorder which majorly targets retinal tissues and contributes to vision loss in diabetic patients. ${ }^{11}$ Earlier, a study suggested that miR$20 b-5 p$ is up-regulated in DR under diabetic conditions. ${ }^{11}$ However, considering miR-20b-5p has a number of targets, studying the functional targets in DR seems to be logical. In the present work, we studied the effects of miR$20 \mathrm{~b}-5 \mathrm{p}$ on diabetic retinopathy endothelial cells and its regulative role on THBS1 gene. We directed our study more toward establishing the importance of THBS1 in diabetic retinopathy. An earlier study suggested that THBS1 can alter the levels of PI3K and Akt and induce changes in development of human gastric cancer. ${ }^{26}$ Hence we speculated that THBS1 can arbitrate the VEGF/Akt/ PI3K cascade and alter pathological changes during diabetic retinopathy. Ultimately, our findings suggested that suppression of miR-20b-5p halted angiogenesis and proliferation of endothelial cells by blocking the VEGF/Akt/ PI3K cascade through over-expression of THBS1.
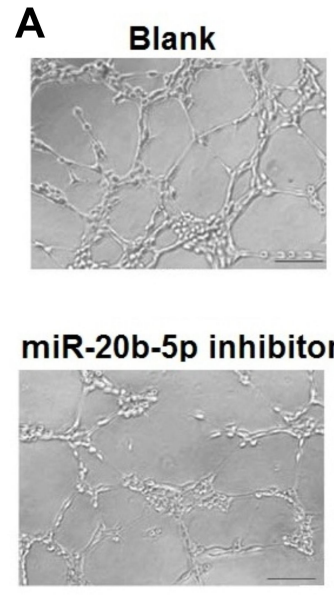

NC

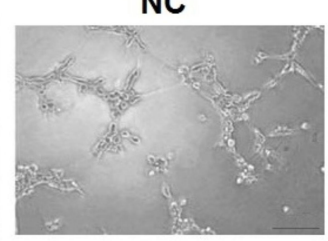

SIRNA-THBS 1

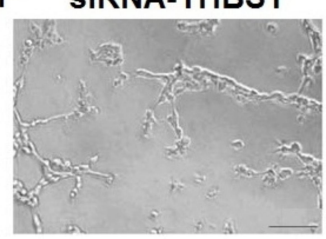

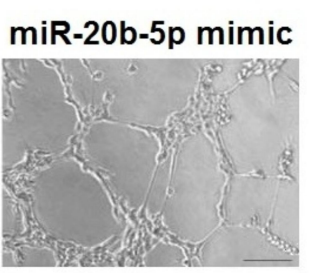

miR-20b-5p inhibitor + siRNA-THBS1

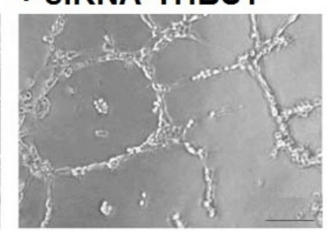

B

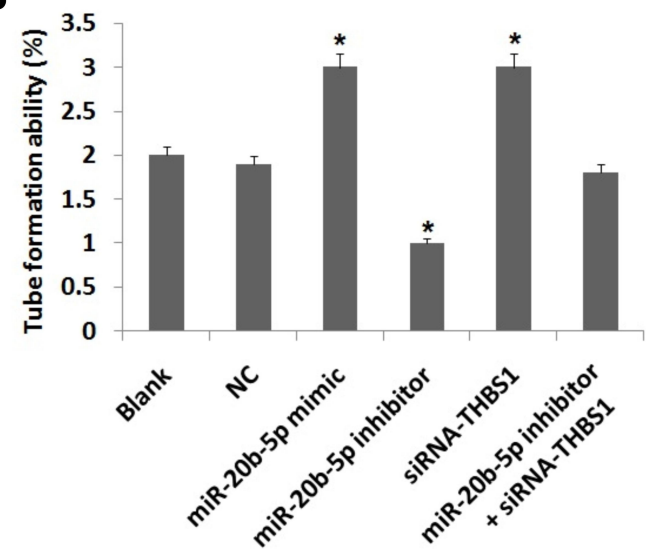

Figure 6 Over-expression of miR-20b-5p or suppression of Thrombospondin I (THBSI) gene promotes tube formation in endothelial cells of diabetic retinopathy rats. (A) Results of tube formation assay post-transfection. (B) Quantitative results of tube formation assay in endothelial cells in diabetic retinopathy rats post-transfection, *P< 0.05 compared to blank group. The results are mean \pm standard deviation. 


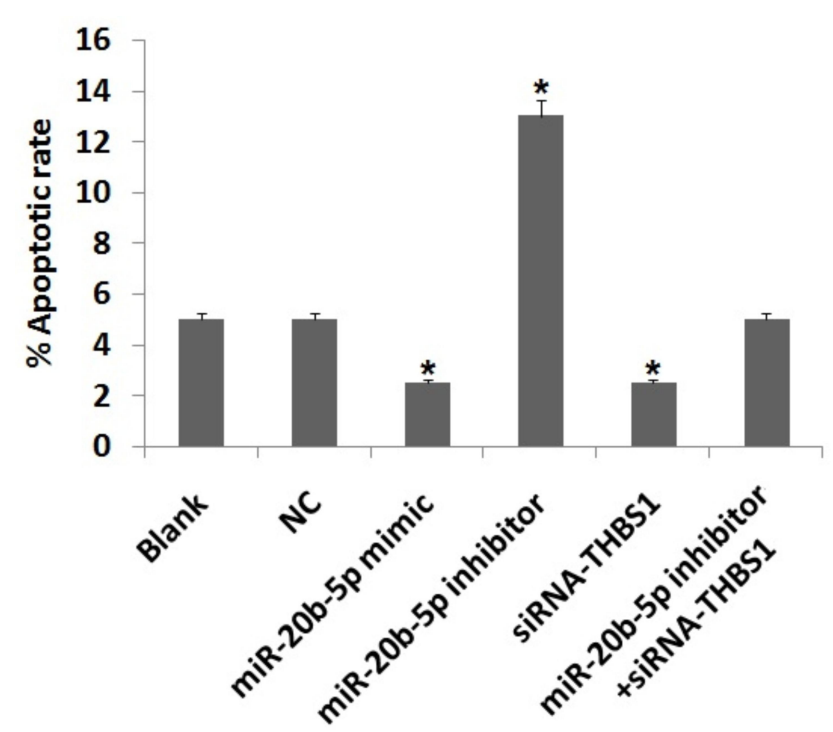

Figure 7 Over-expression of miR-20b-5p or suppression of Thrombospondin I (THBSI) gene suppresses apoptosis in endothelial cells of diabetic retinopathy rats. Results of flow cytometry show percentage of apoptotic cells. $* \mathrm{P}<0.05$ compared to blank. The results are mean \pm standard deviation.

The results suggested that expression of miR-20b-5p was up-regulated in diabetic retinopathy rats showing activation of $\mathrm{VEGF} / \mathrm{Akt} / \mathrm{PI} 3 \mathrm{~K}$ signaling cascade. Also, we proved that over-expression of miR-20b-5p can encourage proliferation of endothelial cells but suppress their apoptosis. Recent findings have demonstrated that deregulation of various miRs such as miR-126 and miR-195 could alter various processes in diabetic retinopathy. ${ }^{27}$ Earlier, it was reported that exosomal expression of miR-20b-5p is elevated in diabetic condition (Type-2) which impaired action of insulin in human skeletal muscles. ${ }^{28}$ miR-20b-5p has been reported to function as tumor suppressor in renal cell carcinoma via regulating cell migration, proliferation, and apoptosis. ${ }^{29}$ A study suggested that suppression of PI3K/Akt/mTOR signaling cascade contributed to improving diabetic retinopathy by decreasing the cell viability via destructing the function of Akt. ${ }^{30}$ VEGF can upregulate the activity of $\mathrm{PI} 3 \mathrm{~K} / \mathrm{Akt}$ signaling cascade, ${ }^{30}$ also, VEGF interferes in the process of angiogenesis in progression of DR. ${ }^{31}$ In the early stages of DR, the VEGF mediated renin angiotensin system leads to increased vascular permeability. ${ }^{32}$ Down-regulation of ICAM-1, NO, and TNF- $\alpha$ leads to decreased inflammation of the retina and also leads to weakening of the blood retinal barrier function contributing to development of DR. ${ }^{4}$ VEGF has also been reported to induce the levels of eNOS in the PI3K/Akt/VEGF/eNOS cascade responsible for synthesis of nitric oxide. ${ }^{33}$ The studies hence confirm that
VEGF/Akt/PI3K can encourage production of nitric oxide affecting progression of diabetic retinopathy. ${ }^{33}$ In the latter part of the study, we proved that expression levels of THBS1 were suppressed in retina of diabetic rats, which was evidenced postendothelial cells were transfected with miR-20b-5p mimic and THBS1 siRNA, which resulted in suppression of THBS1 in addition to upregulation of $\mathrm{VEGF} / \mathrm{Akt} / \mathrm{PI} \mathrm{K}$ cascade. The suppression in expression of THBS1 by siRNA resulted in stimulation and inactivation of proliferation and apoptosis respectively in endothelial cells. Looking into the outcomes of luciferase assay and bioinformatics target prediction, we proved that miR-20b-5p can regulate THBS1 negatively. A study demonstrated that up-regulation of THBS1 can reverse the process of tube formation, whereas suppression can lead to increased cell migration in the process of angiogenesis. ${ }^{34}$ In addition to this, angiogenesis was reported as an important characteristic of diabetic microvascular complication featuring formation of aberrant premature blood vessels in the retinal tissues. ${ }^{35}$ Importantly, THBS1 has been reported to regulate cell proliferation negatively in oral squamous cell carcinoma. ${ }^{36}$

In conclusion, the outcomes of the present work suggested that suppression of miR-20b-5p exerts antiangiogenic effect during development of diabetic retinopathy in rats via inhibiting $\mathrm{VEGF} / \mathrm{Akt} / \mathrm{PI} 3 \mathrm{~K}$ signaling cascade via upregulating the THBS1 gene. The study confirmed the involvement of miR-20b-5p, which can be a promising biomarker in diabetic retinopathy. However, more studies are required to verify the present findings.

\section{Data Sharing Statement}

This can be provided on request.

\section{Ethics Statement}

The animal studies received approval from the animal ethics committee of Heilongjiang Provincial Hospital, Harbin, Heilongjiang 150,036, China. The experiments were done in accordance with ARRIVE guidelines.

\section{Acknowledgments}

The authors are grateful to the staff and management of Heilongjiang Provincial Hospital, Harbin, Heilongjiang. The authors are also grateful to our funding agency scientific research project of Heilongjiang Health and Family Planning Commission for funding this project. 


\section{Author Contributions}

All authors made substantial contributions to conception and design, acquisition of data, or analysis and interpretation of data; took part in drafting the article or revising it critically for important intellectual content; agreed to submit to the current journal; gave final approval of the version to be published; and agree to be accountable for all aspects of the work.

\section{Funding}

This study was supported by Scientific research project of Heilongiiang Health and Family Planning Commission (No: 2018011).

\section{Disclosure}

The authors report no conflicts of interest in this work.

\section{References}

1. Brinchmann-Hansen O, Dahl-Jorgensen K, Hanssen KF, Sandvik L. The response of diabetic retinopathy to 41 months of multiple insulin injections, insulin pumps, and conventional insulin therapy. Arch Ophthalmol. 1988;106(9):1242-1246. doi:10.1001/archopht.198 8.01060140402041

2. Xie MY, Yang Y, Liu P, Luo Y, Tang SB. 5-aza-2'-deoxycytidine in the regulation of antioxidant enzymes in retinal endothelial cells and rat diabetic retina. Int J Ophthalmol. 2019;12(1):1-7. doi:10.18240/ ijo.2019.01.01

3. Obeid A, Su D, Patel SN, et al. Outcomes of eyes lost to follow-up with proliferative diabetic retinopathy that received panretinal photocoagulation versus intravitreal anti-vascular endothelial growth factor. Ophthalmology. 2019;126(3):407-413. doi:10.1016/j. ophtha.2018.07.027

4. Mei X, Zhang T, Ouyang H, Lu B, Wang Z, Ji L. Scutellarin alleviates blood-retina-barrier oxidative stress injury initiated by activated microglia cells during the development of diabetic retinopathy. BiochemPharmacol. 2019;159:82-95.

5. Stitt AW, Lois N, Medina RJ, Adamson P, Curtis TM. Advances in our understanding of diabetic retinopathy. Clin Sci (Lond). 2013;125:1-17. doi:10.1042/CS20120588

6. Karali M, Manfredi A, Puppo A, et al. MicroRNA-restricted transgene expression in the retina. PLoS One. 2011;6:e22166. doi:10.1371/journal.pone.0022166

7. Wang J, Zhang J, Chen X, et al. miR-365 promotes diabetic retinopathy through inhibiting Timp3 and increasing oxidative stress. Exp Eye Res. 2018;168:89-99. doi:10.1016/j.exer.2017.11.006

8. Platania CBM, Maisto R, Trotta MC, et al. Retinal and circulating miRNA expression patterns in diabetic retinopathy: an in silico and in vivo approach. $B r \quad J$ Pharmacol. 2019;176(13):2179-2194. doi:10.1111/bph.14665

9. Lazzara F, Trotta MC, Platania CBM, et al. Stabilization of HIF-1 $\alpha$ in human retinal endothelial cells modulates expression of miRNAs and proangiogenic growth factors. Front Pharmacol. 2020;11:1063. doi:10.3389/fphar.2020.01063

10. Maisto R, Trotta MC, Petrillo F, et al. Resolvin D1 modulates the intracellular VEGF-related miRNAs of retinal photoreceptors challenged with high glucose. Front Pharmacol. 2020;11:235. doi:10.3389/fphar.2020.00235
11. Zhu K, Hu X, Chen H, et al. Downregulation of circRNA DMNT3B contributes to diabetic retinal vascular dysfunction through targeting miR-20b-5p and BAMBI. EBioMedicine. 2019;49:341-353. doi:10.1016/j.ebiom.2019.10.004

12. Guo J, Miao Y, Xiao B, et al. Differential expression of microRNA species in human gastric cancer versus non-tumorous tissues. $J$ Gastroenterol Hepatol. 2009;24(4):652-657. doi:10.1111/j.14401746.2008.05666.x

13. Coskun E, Neumann M, Schlee C, et al. MicroRNA profiling reveals aberrant microRNA expression in adult ETP-ALL and functional studies implicate a role for miR-222 in acute leukemia. Leuk Res. 2013;37(6):647-656. doi:10.1016/j.leukres.2013.02.019

14. Perez-Rivas LG, Jerez JM, Carmona R, et al. A microRNA signature associated with early recurrence in breast cancer. PLoS One. 2014;9 (3):e91884. doi:10.1371/journal.pone.0091884

15. Yamaguchi $\mathrm{T}$, Iijima $\mathrm{T}$, Wakaume $\mathrm{R}$, et al. Underexpression of miR-126 and miR-20b in hereditary and nonhereditary colorectal tumors. Oncology. 2014;87(1):58-66. doi:10.1159/000363303

16. Peng L, Li S, Li Y, et al. Regulation of BTG3 by microRNA-20b-5p in non-small cell lung cancer. Oncol Lett. 2019;18(1):137-144. doi:10.3892/ol.2019.10333

17. Li S, Li H, Xu Y, Lv X. Identification of candidate biomarkers for epithelial ovarian cancer metastasis using microarray data. Oncol Lett. 2017;14(4):3967-3974. doi:10.3892/ol.2017.6707

18. Chen Z, Liu G, Xiao Y, Lu P. Adrenomedullin22-52 suppresses high-glucose- induced migration, proliferation, and tube formation of human retinal endothelial cells. Mol Vis. 2014;20:259-269.

19. Suganthalakshmi B, Anand R, Kim R, et al. Association of VEGF and eNOS gene polymorphisms in type 2 diabetic retinopathy. Mol Vis. 2006;12:336-341.

20. Zhang Y, Wang L, Zhang Y, et al. Nogo-B promotes angiogenesis in proliferative diabetic retinopathy via VEGF/PI3K/Akt pathway in an autocrine manner. Cell Physiol Biochem. 2017;43:1742-1754.

21. He M, Long P, Yan W, et al. ALDH2 attenuates early-stage STZ-induced aged diabetic rats retinas damage via Sirt1/Nrf2 pathway. Life Sci. 2018;215:227-235. doi:10.1016/j.lfs.2018.10.019

22. Kashchenko NI, Chirikova NK, Olennikov DN. Agrimoniin, an active ellagitannin from comarumpalustre herb with anti-_glucosidase and antidiabetic potential in streptozotocin-induced diabetic rats. Molecules. 2017;22:E73. doi:10.3390/molecules22010073

23. Chen H, Sharp BM. Content-rich biological network constructed by mining PubMed abstracts. BMC Bioinform. 2004;5:147. doi:10.1186/ 1471-2105-5-147

24. Piñero J, Bravo À, Queralt-Rosinach N, et al. DisGeNET:a comprehensive platform integrating information on human disease-associated genes and variants. Nucleic Acids Res. 2017;45 (D1):D833-D839. doi:10.1093/nar/gkw943

25. Li Y, Turpin CP, Wang S. Role of thrombospondin 1 in liver diseases. Hepatol Res. 2017;47(2):186-193. doi:10.1111/hepr.12787

26. Huang T, Wang L, Liu D, et al. FGF7/FGFR2 signal promotes invasion and migration in human gastric cancer through upregulation of thrombospondin-1. Int $J$ Oncol. 2017;50(5):1501-1512. doi:10.3892/ijo.2017.3927

27. McAuley AK, Dirani M, Wang JJ, Connell PP, Lamoureux EL, Hewitt AW. A genetic variant regulating miR-126 is associated with sight threatening diabetic retinopathy. Diab Vasc Dis Res. 2015;12:133-138. doi:10.1177/1479164114560160

28. Katayama M, Wiklander OPB, Fritz T, et al. Circulating exosomal miR-20b-5p is elevated in type 2 diabetes and could impair insulin action in human skeletal muscle. Diabetes. 2019;68(3):515-526. doi: $10.2337 / \mathrm{db} 18-0470$

29. Li Y, Chen D, Jin L, et al. MicroRNA-20b-5p functions as a tumor suppressor in renal cell carcinoma by regulating cellular proliferation, migration and apoptosis. Mol Med Rep. 2016;13(2):1895-1901. doi: $10.3892 / \mathrm{mmr} .2015 .4692$ 
30. Fang S, Ma X, Guo S, Lu J. MicroRNA-126 inhibits cell viability and invasion in a diabetic retinopathy model via targeting IRS-1. Oncol Lett. 2017;14:4311-4318. doi:10.3892/ol.2017.6695

31. Witmer AN, Blaauwgeers HG, Weich HA, Alitalo K, Vrensen GF, Schlingemann RO. Altered expression patterns of VEGF receptors in human diabetic retina and in experimental VEGF-induced retinopathy in monkey. Invest Ophthalmol Vis Sci. 2002;43:849-857.

32. Kim JH, Kim JH, Yu YS, Cho CS, Kim KW. Blockade of angiotensin ii attenuates VEGF-mediated blood-retinal barrier breakdown in diabetic retinopathy. J Cereb Blood Flow Metab. 2008;29:621-628. doi:10.1038/jcbfm.2008.154

33. Ma J, Sawai H, Ochi N, et al. PTEN regulates angiogenesis through PI3K/ Akt/VEGF signaling pathway in human pancreatic cancer cells. Mol Cell Biochem. 2009;331:161-171. doi:10.1007/s11010-009-0154-x
34. Shih HJ, Chen CL, Torng PL. IGFBP3 inhibits angiogenesis through intracellular regulation of THBS1 expression. Am $J$ Cancer Res. 2020;10(6):1728-1744.

35. Cheng R, Ma JX. Angiogenesis in diabetes and obesity. Rev Endocr Metab Disord. 2015;16:67-75.

36. Pal SK, Nguyen CT, Morita KI, et al. THBS1 is induced by TGFB1 in the cancer stroma and promotes invasion of oral squamous cell carcinoma. J Oral Pathol Med. 2016;45(10):730-739. doi:10.1111/ jop. 12430

Diabetes, Metabolic Syndrome and Obesity: Targets and Therapy

\section{Publish your work in this journal}

Diabetes, Metabolic Syndrome and Obesity: Targets and Therapy is an international, peer-reviewed open-access journal committed to the rapid publication of the latest laboratory and clinical findings in the fields of diabetes, metabolic syndrome and obesity research. Original research, review, case reports, hypothesis formation, expert opinion and commentaries are all considered for publication. The manuscript management system is completely online and includes a very quick and fair peer-review system, which is all easy to use. Visit http://www.dovepress.com/testimonials.php to read real quotes from published authors. 